\title{
Connecting the Higgs potential and primordial black holes
}

\author{
De-Chang Dai $\odot,{ }^{1,2}$ Ruth Gregory $\odot,{ }^{3,4}$ and Dejan Stojkovic ${ }^{5}$ \\ ${ }^{1}$ Center for Gravity and Cosmology, School of Physics Science and Technology, Yangzhou University, \\ 180 Siwangting Road, Yangzhou City, Jiangsu Province 225002, People's Republic of China \\ ${ }^{2}$ CERCA/Department of Physics/ISO, Case Western Reserve University, \\ Cleveland, Ohio 44106-7079, USA \\ ${ }^{3}$ Centre for Particle Theory, Durham University, South Road, Durham DH1 3LE, United Kingdom \\ ${ }^{4}$ Perimeter Institute, 31 Caroline Street, Waterloo, Ontario N2L 2Y5, Canada \\ ${ }^{5}$ HEPCOS, Department of Physics, SUNY at Buffalo, Buffalo, New York 14260-1500, USA
}

(Received 6 September 2019; accepted 3 June 2020; published 15 June 2020)

\begin{abstract}
It was recently demonstrated that small black holes can act as seeds for nucleating decay of the metastable Higgs vacuum, dramatically increasing the tunneling probability. Any primordial black hole lighter than $4.5 \times 10^{14} \mathrm{~g}$ at formation would have evaporated by now, and in the absence of new physics beyond the standard model, would therefore have entered the mass range in which seeded decay occurs, however, such true vacuum bubbles must percolate in order to completely destroy the false vacuum; this depends on the bubble number density and the rate of expansion of the universe. Here, we compute the fraction of the universe that has decayed to the true vacuum as a function of the formation temperature (or equivalently, mass) of the primordial black holes, and the spectral index of the fluctuations responsible for their formation. This allows us to constrain the mass spectrum of primordial black holes given a particular Higgs potential and conversely, should we discover primordial black holes of definite mass, we can constrain the Higgs potential parameters.
\end{abstract}

DOI: $10.1103 /$ PhysRevD.101.125012

\section{INTRODUCTION}

One of the most fascinating implications of the measurement of the Higgs mass at the LHC [1,2] is that the standard model vacuum appears to be metastable [3-11]. Initially, this was not thought to be a problem for our universe, as standard techniques for computing vacuum decay [12-15] indicated that the half-life was many order of magnitude greater the age of the universe. However, vacuum decay represents a first order phase transition, and in nature these typically proceed via catalysis: a seed or impurity acts as a nucleus for a bubble of the new phase to form. In [16-20], the notion that a black hole could act as such a seed was explored, with the finding that black holes can dramatically shorten the lifetime of a metastable vacuum (see also [21-28]). Interestingly, before the discovery of the Higgs particle, the electroweak phase transition was usually described as a second order transition, and in [29] the idea that the usual second order

Published by the American Physical Society under the terms of the Creative Commons Attribution 4.0 International license. Further distribution of this work must maintain attribution to the author(s) and the published article's title, journal citation, and DOI. Funded by SCOAP ${ }^{3}$. electroweak phase transition might be followed by a first order phase transition was explored.

For a black hole to seed vacuum decay, we must be sure that the half-life for decay is less than the evaporation rate of the black hole. This means that the branching ratio of tunnelling to decay must be greater than one. In $[18,19]$ this was found to occur for black holes of order $10^{6-9} M_{p}$ or so, by which point the half-life for decay is of order $10^{-23} \mathrm{~s}$. Clearly this process is not relevant for astrophysical black holes, however, it has been hypothesised that there exist very light black holes, formed from extreme density fluctuations in the early universe [30-32] dubbed primordial black holes. Such black holes have been proposed as a source for dark matter [33], and although this has now been ruled out [34], they could still constitute a component of the dark matter of the universe. Indeed, it has even been proposed that the Higgs vacuum instability could generate primordial black holes in the early universe [35].

Given that we are in a current metastable Higgs vacuum, we can be sure that there has been no primordial black hole that has evaporated in our past lightcone, however, how strong a constraint on primordial black holes can we place? For the universe to have decayed, the black hole must not only have evaporated sufficiently to reach the mass range in which catalysis spectacularly dominates, but the consequent bubble (or bubbles) of true vacuum must have percolated 
to engulf the current Hubble volume. Thus, this is a statement about the relative volume in the percolated bubble, which is itself a statement on the primordial black hole density and mass. In this paper, we draw together all these aspects of the problem, linking the primordial black hole spectral index and formation epoch to the standard model parameters.

The outline of the paper is as follows. In Sec. II we review the physics of the Higgs vacuum decay in the presence of gravity. In Sec. III we relate the primordial black hole masses that can trigger vacuum decay with the parameters in the effective Higgs potential. In Sec. IV we put this scenario in the cosmological context: Every black hole that can trigger the vacuum decay will create a bubble of true vacuum. These bubbles then expand with the speed of light, but their number density decreases due to the expansion of the universe. For a successful phase transition, the bubbles have to percolate, so we define a quantity $\mathcal{P}$, which represents the portion of the universe that has already transitioned to the new vacuum. For $\mathcal{P} \geq 1$, the universe would be destroyed, thus the associated range of parameters is excluded. We summarize and discuss our findings in Sec. V.

\section{FALSE VACUUM DECAY WITH BLACK HOLES}

The high energy effective Higgs potential has been determined by a two-loop calculation in the standard model as [7]

$$
V(\phi)=\frac{1}{4} \lambda_{\mathrm{eff}}(\phi) \phi^{4},
$$

where $\lambda_{\text {eff }}(\phi)$ is the effective coupling constant that runs with scale. We now review the calculations in [19], adopting the same conventions. The running of the coupling constant can be excellently modelled over a large range of scales by the three parameter fit:

$$
\lambda_{\text {eff }}=\lambda_{*}+b\left(\ln \frac{\phi}{M_{p}}\right)^{2}+c\left(\ln \frac{\phi}{M_{p}}\right)^{4},
$$

where $M_{p}^{-2}=8 \pi G$. By fitting the two-loop calculation with a simple analytic form, we can easily investigate not only the standard model, but also beyond the standard model potentials, allowing us to explore possible future corrections to the standard model results.

The Higgs potential supports a first order phase transition mediated via nucleation of bubbles of new vacuum inside the old, false, vacuum. The nucleation rate in the presence of gravity is determined by a saddle point "bounce" solution of the Euclidean (signature,,,++++ ) action:

$S_{E}=\int_{\mathcal{M}}\left[-\frac{1}{16 \pi G} \mathcal{R}+\left(\frac{1}{2} g^{a b} \partial_{a} \phi \partial_{b} \phi+V(\phi)\right)\right] \sqrt{-g} d^{4} x$.
The spacetime geometry is taken to have $S O(3) \times U(1)$ symmetry, in other words, it is spherically symmetric "around" the black hole, and has time translation symmetry along the Euclidean time direction, $\tau$ :

$d s^{2}=f(r) e^{2 \delta(r)} d \tau^{2}+\frac{d r^{2}}{f(r)}+r^{2}\left(d \theta^{2}+\sin ^{2} \theta d \varphi^{2}\right)$,

with

$$
f(r)=1-\frac{2 G \mu(r)}{r} .
$$

We can think of $\mu(r)$ as the local mass parameter, however caution must be used in pushing this analogy. For an asymptotic vacuum of $\Lambda=0$, then $\mu(\infty)$ truly is the Arnowitt-Deser-Misner (ADM) mass of the black hole, however, locally, $\mu$ also includes the effect of any vacuum energy: for a pure Schwarzschild-(A)dS solution, $\mu(r)=M+\Lambda r^{3} / 6 G$. Since we are interested in seeding the decay of our current Standard Model (SM) vacuum, we will take $\Lambda_{+}=0$, so that the asymptotic value of $\mu$ is indeed the seed black hole mass, $M_{+}$, responsible for triggering the phase transition. The remnant mass, which is a leftover from the seed black hole after some of its energy is invested into the bubble formation, may not be precisely $\mu\left(r_{h}\right)$, however, since we will be interested only in the area of the remnant black hole horizon, it turns out that $\mu\left(r_{h}\right)$ is in fact the desired quantity.

The Higgs and gravitational field equations of motion are

$$
\begin{gathered}
f \phi^{\prime \prime}+f^{\prime} \phi^{\prime}+\frac{2}{r} f \phi^{\prime}+\delta^{\prime} f \phi^{\prime}-V_{, \phi}=0 \\
\mu^{\prime}=4 \pi r^{2}\left(\frac{1}{2} f \phi^{\prime 2}+V\right) \\
\delta^{\prime}=4 \pi G r \phi^{\prime 2},
\end{gathered}
$$

where $V_{, \phi} \equiv \partial V / \partial \phi$. The black hole horizon is at $r=r_{h}$, at which $f\left(r_{h}\right)=0$. We have to solve these equations of motion numerically in order to get the function $\phi(r)$, and to do this, we start from the horizon $r_{h}$ with a particular remnant parameter, $r_{h}=2 G \mu_{-}$, and some value for the Higgs field $\phi_{h}$. At the horizon therefore the fields satisfy the boundary conditions

$$
\begin{aligned}
\mu\left(r_{h}\right) & =\mu_{-}, \quad \delta\left(r_{h}\right)=0 \\
\phi^{\prime}\left(r_{h}\right) & =\frac{r_{h} V_{, \phi}\left(\phi_{h}\right)}{1-8 \pi G r_{h}^{2} V\left(\phi_{h}\right)},
\end{aligned}
$$

and as $r \rightarrow \infty$,

$$
\lim _{r \rightarrow \infty} \phi(r) \rightarrow 0, \quad M_{+}=\lim _{r \rightarrow \infty} \mu(r) .
$$

We use a shooting method starting at $r_{h}$ with $\phi=\phi_{h}$ and integrate out, altering $\phi_{h}$ until a solution is obtained with $\phi$ 
tending to 0 for very large values of $r$. In practice, rather than setting the asymptotic mass $\mu(\infty)=M_{+}$, we set the initial (remnant) value of $\mu_{-}$and deduce the seed mass from (8), repeating the integration for a range of values of $\mu_{-}$. We then determine $\mu_{-}\left(M_{+}\right)$by inversion.

The decay rate of the Higgs vacuum, $\Gamma_{D}$, is then determined by computing the difference in entropy between the seed and remnant black holes:

$$
\Gamma_{D}=\left(\frac{B}{2 \pi}\right)^{1 / 2}\left(G M_{+}\right)^{-1} e^{-B}
$$

where

$$
B=\frac{M_{+}^{2}-\mu_{-}^{2}}{2 M_{p}^{2}}
$$

As pointed out in [17-19], a black hole can also radiate and lose mass, eventually disappearing in Hawking radiation, at a rate initially estimated by Page [36], see also [37-41]:

$$
\Gamma_{H}=3.6 \times 10^{-4}\left(G^{2} M_{+}^{3}\right)^{-1} .
$$

Thus, we define the branching ratio between the tunneling and evaporation rate as

$$
\frac{\Gamma_{D}}{\Gamma_{H}}=43.8 \frac{M_{+}^{2}}{M_{p}^{2}} B^{1 / 2} e^{-B}
$$

This equation contains all the information we need. In the next two sections, we will study the consequences of the gravitationally induced false Higgs vacuum decay.

\section{THE VACUUM DECAY RATE AND THE HIGGS EFFECTIVE POTENTIAL}

If the branching ratio given by Eq. (12) is larger than one, then the tunneling rate is faster than evaporation rate, and the black hole can catalyze false vacuum decay. Note that the branching ratio depends on three parameters: $M_{+}, \lambda_{*}$, and $b$ : fitting the form of $\lambda_{\text {eff }}$ in (2) to the standard model value at the electroweak scale fixes $c$ in terms of $\lambda_{*}$ and $b$, and $M_{+}$is the primordial black hole seed.

Let us first illustrate the results for some sample choices of the potential parameters. If we set $\lambda_{*}=-0.004$, $b=1.5 \times 10^{-5}, c=0$, then Fig. 1 shows that the branching ratio is larger than one for

$$
M_{p} \lesssim M_{+} \lesssim 10^{6} M_{p}
$$

This means that primordial black holes with masses within this range can initiate Higgs vacuum decay for the associated values of the Higgs potential parameters. A black hole mass with the lifetime of the current age of the

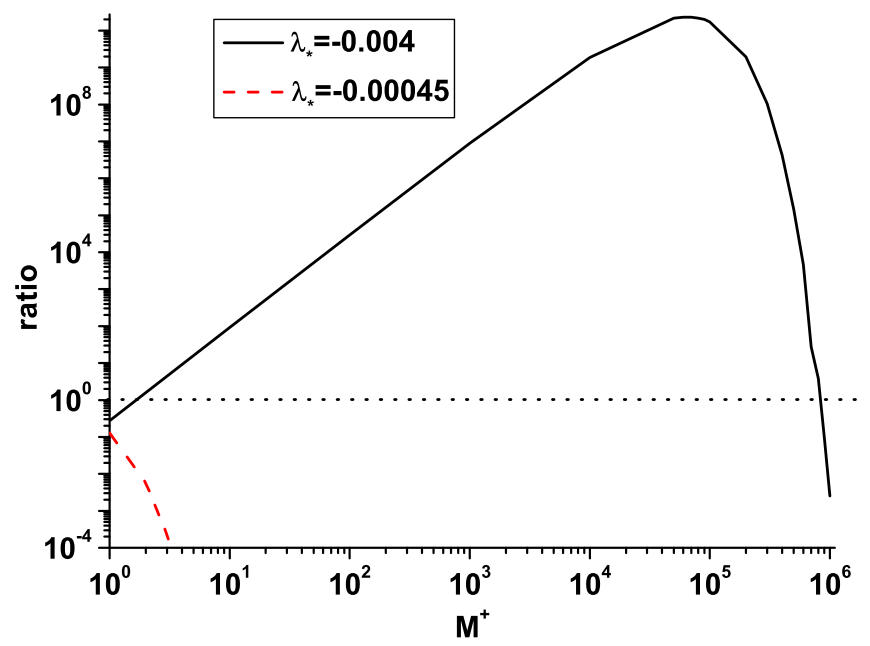

FIG. 1. The branching ratio between the tunneling and evaporation rate as a function of the seed black hole mass, $M_{+}$, for some sample choices of the potential parameters. The unit branching ratio, $\Gamma_{D} / \Gamma_{H}=1$, is labeled by the dotted line. We set $b=1.5 \times 10^{-5}, c=0 . M_{+}$is given in units of $M_{p}$.

universe is approximately $4.5 \times 10^{14}$ grams, meaning that all black holes lighter than this value would have already evaporated. Along the way, they will inevitably end up in the range given by Eq. (13). This however does not automatically imply that all the primordial black holes lighter than $4.5 \times 10^{14}$ grams are excluded for this choice of parameters. To destroy the universe the bubbles of the true vacuum have to percolate, which takes time. We will study this in the next section.

The same Fig. 1 indicates that if we set $\lambda_{*}=-0.00045$, and keep $b=1.5 \times 10^{-5}$ and $c=0$, then the branching ratio is always smaller than one (these values are not consistent with a pure standard model effective coupling, however, indicate the principle of model dependence of the branching ratio). In that case, the primordial black holes of any mass (i.e., $M_{p}<M_{+}<\infty$ ) cannot stimulate the false vacuum to decay into true vacuum, and our universe is safe. We excluded the black hole seed masses less than $M_{p}$ from the discussion, as the semiclassical approximation used in computing the decay rate is no longer expected to be valid at the Planck scale, where presumably a full theory of quantum gravity is required.

It is now instructive to systematically analyze the range of parameters for the effective coupling (2). Figure 2 shows the threshold curve $\frac{\Gamma_{D}}{\Gamma_{H}}=1$ in $\left(b, \lambda_{*}\right)$ parameter space for two values of the parameter $c$. The region of parameter space with $\frac{\Gamma_{D}}{\Gamma_{H}}<1$, for which the universe is safe, is above the curve. Below the curve, the branching ration will be greater than one for some range of black hole masses [similar to that shown in Eq. (13)] below the quantum gravity scale. This range is different for differing $\lambda_{*}, b$, and $c$ (so not easy to plot) however, it can easily calculated by substituting the concrete values for $\lambda_{*}, b$, and $c$, in Eq. (12). 


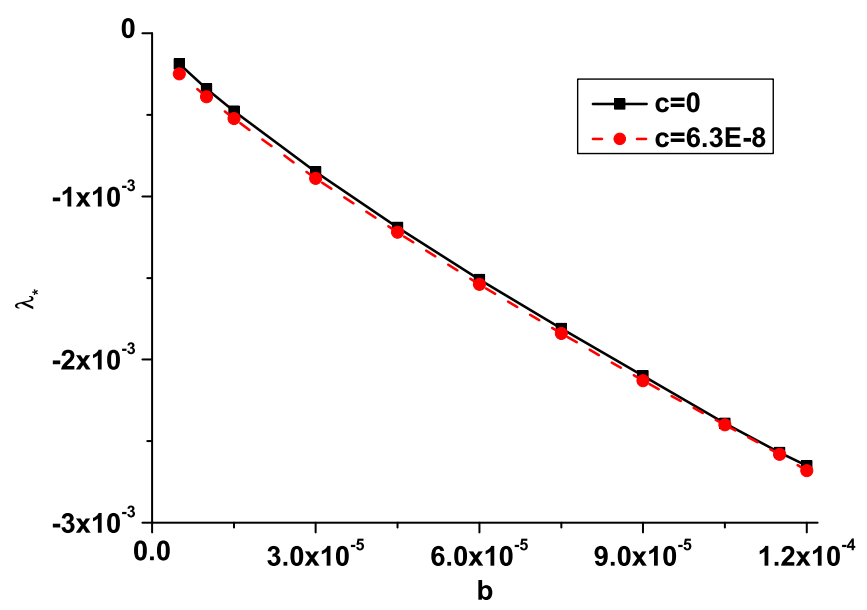

FIG. 2. A plot of the line $\frac{\Gamma_{D}}{\Gamma_{H}}=1$ in $\left(b, \lambda_{*}\right)$ parameter space for the values $c=0,6.3 \times 10^{-8}$ as labeled. Above the curve, the branching ratio $\frac{\Gamma_{D}}{\Gamma_{H}}$ is always less than one for any value of the seed black hole mass. Below the curve, there is always a range of the black hole masses for which the branching ratio $\frac{\Gamma_{D}}{\Gamma_{H}}$ is greater than one. The dependence on $c$ is minimal.

The boundary with $c=6.3 \times 10^{-8}$ is lower than that with $c=0$ because of the contribution from the quartic terms in the Higgs potential. However, numerical experiments indicate that the curves do not change significantly as we vary the parameter $c$.

According to [19] the standard model parameter space corresponding to the allowed range of top quark mass $(172-174 \mathrm{GeV})$ is $1.2 \times 10^{-5} \lesssim b \lesssim 1.4 \times 10^{-5}$ and $-0.02 \lesssim \lambda_{*} \lesssim-0.007$. Unfortunately, this lies inside the potentially dangerous $\frac{\Gamma_{D}}{\Gamma_{H}}>1$ region. If some new physics beyond the standard model modifies the Higgs potential, then we can potentially move outside of these parameter ranges. In the best case, the Higgs vacuum gets stabilized and the first order phase transition disappears altogether from the discussion. However, there is also a potentially dangerous region where beyond the standard model corrections give a "safe" long false vacuum lifetime in the absence of black holes, yet unacceptably short in their presence. Thus, one can always tension new beyond the standard model physics with the existence of the primordial black holes of a certain mass.

\section{PRIMORDIAL BLACK HOLE MASSES AND PERCOLATING BUBBLES}

In the previous section, we saw that any primordial black hole that had enough time to evaporate sufficiently to fit into an appropriate mass range for the corresponding choice of the parameters $\lambda_{*}$ and $b$, could initiate false vacuum decay. The bubbles of true vacuum then expand with the speed of light, but the background universe expands as well. Successful completion of the first order phase transition depends on the number density of the created bubbles. In our scenario, every black hole that can initiate the false vacuum decay will create a bubble, so the number of the bubbles is equal to the number of such primordial black holes. Thus, whether the initiated vacuum decay can be completed crucially depends on the production mechanism and age of the universe when the primordial black holes were formed.

It is usually assumed that primordial black holes are produced by density fluctuations caused by oscillations of some (scalar) field. If density fluctuations are large enough [42-47], the whole causally connected region (i.e., the horizon volume at some time) collapses and forms a black hole. The horizon mass in a radiation-dominated universe (in units of grams) is

$$
M_{H} \cong 10^{18}\left(\frac{10^{7} \mathrm{GeV}}{T}\right)^{2} \mathrm{~g}
$$

where $T$ is the temperature of radiation. Obviously, the earlier the black holes are formed, the lighter they are, hence their lifetime is shorter. Their lifetime is given as [36-41].

$$
\tau_{\text {evap }}=4.99 \times 10^{-44}\left(\frac{M}{M_{P}}\right)^{3} \mathrm{~s} .
$$

Black holes of mass $M \gtrsim 4.5 \times 10^{14} \mathrm{~g}$ have a lifetime greater than $1.38 \times 10^{10}$ years, or the age of the universe. Therefore only lighter primordial black holes will have the potential to destroy the universe, based on the SM parameter ranges. We focus mostly on these lighter black holes which, according to Eq. (14), are created at temperatures higher than $T_{F} \gtrsim 4.7 \times 10^{8} \mathrm{GeV}$.

After primordial black holes are formed at $T_{F}$, their number density changes with temperature as

$$
n_{b}(T) \sim \frac{\beta_{\mathrm{i}}}{M_{i}} \rho_{r, i}\left(\frac{T}{T_{F}}\right)^{3} .
$$

where $\beta_{i}$ is the mass fraction of the universe in black holes at formation, while $\rho_{r, i}=\frac{\pi^{2}}{30} g_{F} T_{F}^{4}$ is the radiation energy density at that time, with $g_{F} \approx 100$ being the number of degrees of freedom of radiation species at $T_{F} . M_{F}$ is the mass of the primordial black holes at formation, and we take $M_{F}=M_{H}\left(T_{F}\right)$ as usual. The mass fraction $\beta_{i}$ can be found assuming a Gaussian perturbation spectrum of fluctuations that lead to black hole formation (see e.g., $[42,48,49])$

$$
\beta_{i} \approx \frac{\sigma_{H}\left(T_{F}\right)}{\sqrt{2 \pi} \delta_{\min }} e^{-\frac{\delta_{\min }^{2}}{2 \sigma_{H}^{2}\left(T_{F}\right)}},
$$

The parameter $\delta_{\min } \approx 0.3$ is the minimum density contrast required for black hole creation, while $\sigma_{H}(T)$ is the mass 
variance evaluated at horizon crossing at the temperature $T$ defined as [48]

$$
\sigma_{H}\left(T_{F}\right)=\sigma_{H}\left(T_{0}\right)\left(\frac{M_{H}\left(T_{0}\right)}{M_{H}\left(T_{\text {eq }}\right)}\right)^{\frac{n-1}{6}}\left(\frac{M_{H}\left(T_{\text {eq }}\right)}{M_{H}\left(T_{F}\right)}\right)^{\frac{n-1}{4}} .
$$

Here, $T_{\text {eq }} \approx 0.79 \mathrm{eV}$ is the temperature at the matter/ radiation equilibrium, $T_{0}=2.725 \mathrm{~K}=2.35 \times 10^{-4} \mathrm{eV}$ is the present temperature of the universe, while $n$ is the spectral index of the fluctuations that lead to black hole formation, i.e., $P(k) \propto k^{n}$. Note, the cosmic microwave background data indicate that the value of the spectral index of the inflaton field is $n \approx 1$, however the cosmic microwave background $(\mathrm{CMB})$ data probe the scales between $10^{45}$ and $10^{60}$ times larger than those probed by primordial black holes. It is expected that primordial black holes are formed by fluctuations of fields other than the inflaton (e.g., during phase transitions), and the typical value of $n$ used in this context is between 1.23 and 1.31 [42,49,50]. To normalize Eq. (18) we use the mass variance evaluated at the horizon crossing $\sigma_{H}\left(T_{0}\right)=9.5 \times 10^{-5}$.

We now have all the elements to calculate the black hole abundance for any set of desired parameters. After formation, primordial black holes evaporate, and at some stage of their life they will trigger false vacuum decay. When exactly this will happen depends on the specific parameters of the Higgs potential; we must be above the threshold value of the branching ratio, or in the range of parameters below the curve in Fig. 2, where it is guaranteed that the phase transition will be initiated for some black hole mass range.

To illustrate the procedure, we calculate the excluded primordial black hole parameter space for the example from Sec. II, i.e., for the values of the potential parameters $\lambda_{*}=-0.004, b=1.5 \times 10^{-5}, c=0$. As shown in Sec. II, the branching ratio is larger than one for the seed black hole masses $M_{p} \lesssim M_{+} \lesssim 10^{6} M_{p}$, therefore all the black holes that have evaporated down to $10^{6} M_{p}$ or less by the present time will trigger false vacuum decay for this set of parameters. We note that this number is effectively the same as the number of the black holes that have evaporated completely by the present time, since it takes only a fraction of the second for a black hole to evaporate from $10^{6} M_{p}$ to zero. For comparison, we will also add black hole masses that correspond to the parameters beyond the standard model.

The scenario is as follows. Suppose that primordial black holes are formed at a temperature $T_{F}$ with some initial mass $M_{i}$. They then evaporate until they reach a mass at which vacuum decay is catalyzed $\left(10^{6-9} M_{p}\right.$ for the standard model values, which is essentially equivalent to a complete evaporation, given the scale of the lifetimes involved). For the parameters of the Higgs potential outside of the standard model, these masses could be much higher. At that moment (which depends on the initial black hole mass) they seed vacuum decay and form a bubble of true vacuum that then expands at the speed of light. For a successful phase transition, the bubbles have to percolate, so we compute the overall volume of true vacuum in the expanding universe from the volume of an individual bubble and the number density of black holes.

The present time number density of the bubbles, $n_{\mathrm{b}}\left(T_{0}\right)$, is shown in Fig. 3. It is calculated from Eq. (16) following the procedure outlined above. The present time radius of the bubble depends on the time it was created. If an object (in this case a bubble of true vacuum) is created at a cosmological redshift $Z$, its present age, $t$, is given by

$$
t(Z)=\frac{1}{H_{0}} \int_{0}^{Z} \frac{d z}{E(1+z)}
$$

where

$$
\begin{aligned}
E^{2}=\frac{H^{2}}{H_{0}^{2}}= & \Omega_{m}(1+Z)^{3}+\Omega_{\mathrm{rad}}(1+Z)^{4} \\
& +\Omega_{k}(1+Z)^{2}+\Omega_{\Lambda} .
\end{aligned}
$$

Here $\Omega_{m}, \Omega_{\text {rad }}, \Omega_{k}$ and $\Omega_{\Lambda}$ are the present values of the dark matter, radiation, curvature, and dark energy density respectively. We take their numerical values from Planck results [51], $\Omega_{m}=0.315, \Omega_{\text {rad }}=9.23 \times 10^{-5}, \Omega_{k}=0$ and $\Omega_{\Lambda}=0.684 . H_{0}$ is the present time Hubble parameter, $H_{0}=67.4 \mathrm{~km} \mathrm{~s}^{-1} \mathrm{Mpc}^{-1}$. The connection between the temperature, $T$, scale factor, $a$, and the redshift, $Z$, in an expanding Friedman Robertson Walker universe is $T \propto 1 / a \propto(1+Z)$.

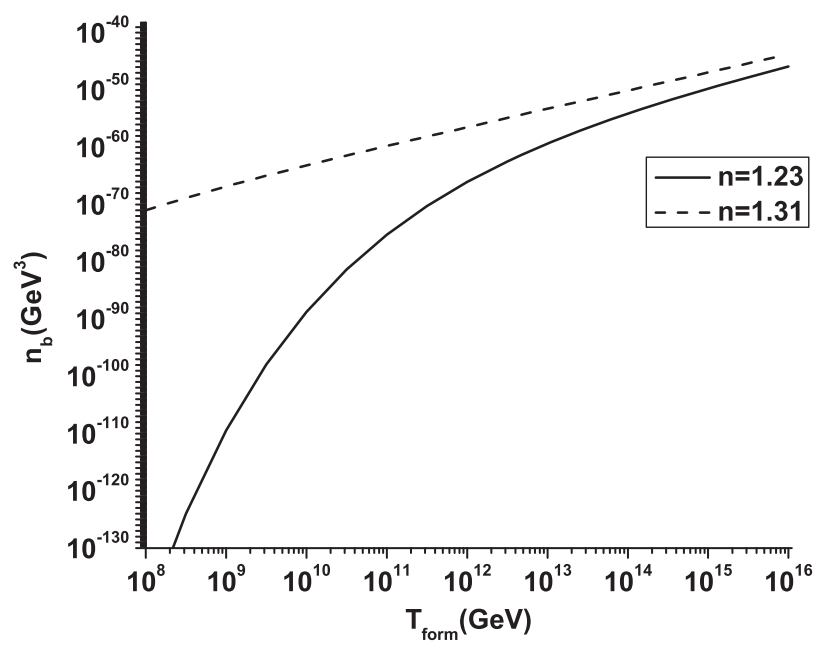

FIG. 3. The present number density of the true vacuum bubbles, plotted as a function of the temperature at which the primordial black holes that seed nucleation are formed. The number density is shown for two values of the spectral density index, $n$, of the perturbations responsible for the primordial black hole creation. 
Since $d r=c d t / a=c d t(1+Z)$, the current physical radius of the true vacuum bubble formed at redshift $Z_{B}$ is

$$
R=\frac{c}{H_{0}} \int_{0}^{Z_{B}} \frac{d z}{E}
$$

where $E$ is given by Eq. (20). The redshift, $Z_{B}$, is calculated at the moment when a black hole of a certain seed mass (formed at the temperature $T_{F}$ ) evaporates enough to fit into the appropriate mass window where it can trigger the false vacuum decay.

Thus, the portion of the universe which is already in the new vacuum at the present time is

$$
\mathcal{P}=\frac{4 \pi}{3} R^{3} n_{b}\left(T_{0}\right)
$$

Figure 4 shows the boundary of the $\mathcal{P}=1$ region. For the range of parameters in the upper part of the plot the universe today is destroyed, since the bubbles percolate.

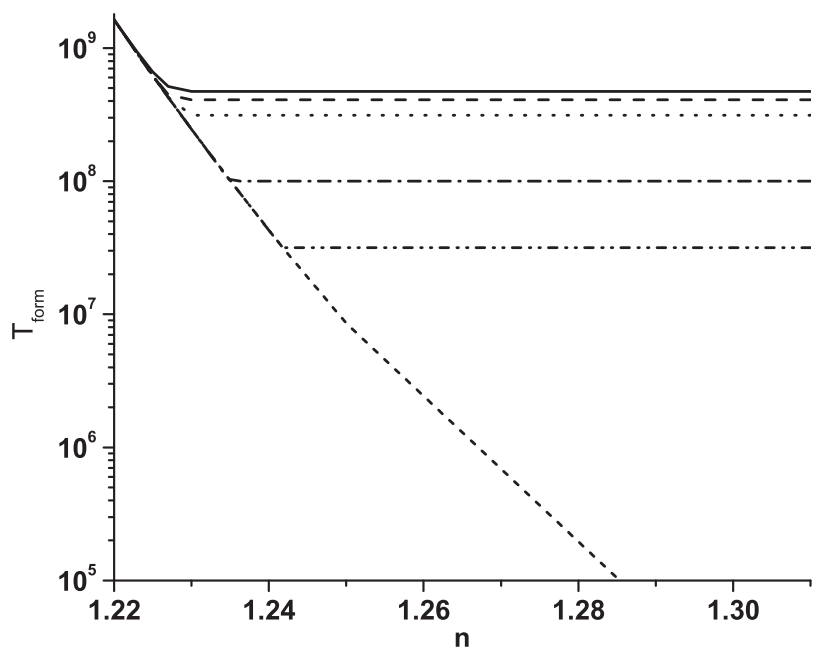

FIG. 4. This plot shows the temperature of the universe at the time of the primordial black hole formation, $T_{F}$, as a function of the spectral index, $n$ for several values of the black hole masses that can trigger the vacuum decay. The lines represent the $\mathcal{P}=1$ value for the portion of the universe which already transitioned to the new vacuum at present time. Above these lines, we have $\mathcal{P}>1$ and the whole universe today would be destroyed. Below these lines we have $\mathcal{P}<1$, and the universe is safe. A black hole of the initial mass $M_{i}$ (determined by the formation temperature $T_{F}$ ) evaporates and triggers the vacuum decay at some lower value $M_{\mathrm{dec}}$. If $M_{i}>M_{\mathrm{dec}}$, the onset of the phase transition is delayed by the time needed for a black hole to enter the window where it can trigger the vacuum decay with $\frac{\Gamma_{D}}{\Gamma_{H}}>1$. The solid line corresponds to the black hole masses $M_{\text {dec }} \ll 5 \times 10^{14}$ g, i.e., $M_{\mathrm{dec}} \approx 0$. The dashed, dot, dashed dot, and dashed dot dot lines correspond to $M_{\text {dec }}$ values of $5 \times 10^{14} \mathrm{~g}, 10^{15} \mathrm{~g}, 10^{16} \mathrm{~g}, 10^{17} \mathrm{~g}$. The short dashed line corresponds to $M_{i}<M_{\mathrm{dec}}$, where such black holes trigger the vacuum decay immediately upon formation.
In contrast, for the range of parameters in the lower part of the plot, the universe is safe, though the primordial black holes may initiate false vacuum decay. We distinguish between the initial black hole mass $M_{i}$ (determined by the formation temperature $T_{F}$ ) which evaporates and triggers the vacuum decay at some lower value $M_{\mathrm{dec}}$, determined by the requirement $\frac{\Gamma_{D}}{\Gamma_{H}}>1$. To show how the results vary with the mass of the black hole that triggers the vacuum decay, we plot the curves corresponding to several values of $M_{\mathrm{dec}}$.

With the help of Eq. (14), we can convert the temperature of the universe at the time of the primordial black hole formation to the primordial black hole mass. This is shown in Fig. 5. We can see that lighter black holes are more dangerous than the more massive ones because they evaporate quickly, form the true vacuum bubbles earlier, and the bubbles have more time to grow. Black holes much heavier than $M \gtrsim 10^{17} \mathrm{~g}$ are not constrained since they did not have enough time to evaporate down to the dangerous range of masses. A potentially interesting range of the parameter space is where $\mathcal{P}$ is nonzero but not too close to one. This would mean that there are a few bubbles in the universe here and there, but they do not yet dominate the universe. This situation is shown in Fig. 6. Since the bubbles expand with the speed of light, if they are far from us, this state could last for billions of years. It would be very interesting to study observational effects of the presence of such bubbles within our horizon.

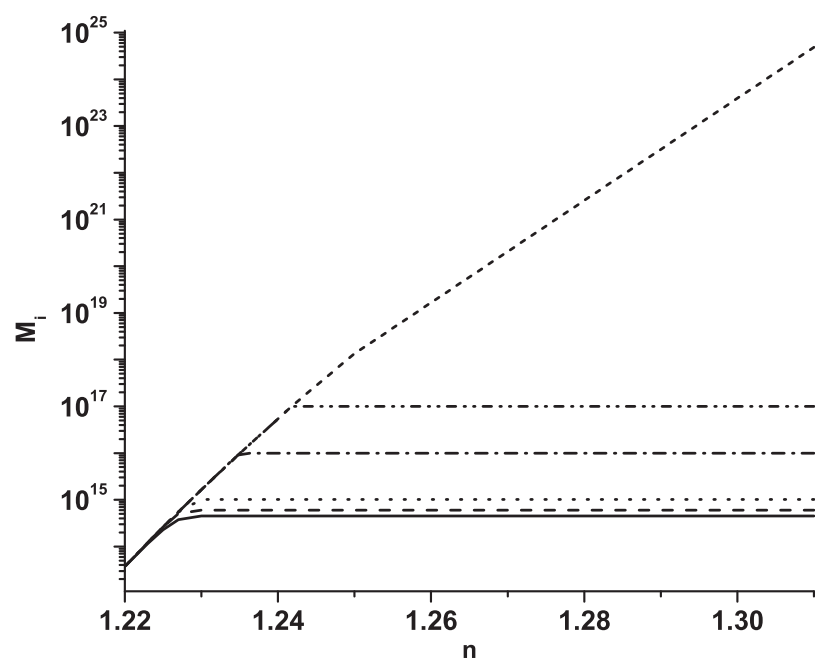

FIG. 5. This plot shows the primordial black hole masses (in grams) at the time of their formation, $M_{i}$, as a function of the spectral index, $n$. The lines represent the $\mathcal{P}=1$ value for the portion of the universe which already transitioned to the new vacuum at present time. Below this line we have $\mathcal{P}>1$ and the whole universe today would be destroyed. Above this line, we have $\mathcal{P}<1$, and the universe is safe. The solid line corresponds to the black hole masses $M_{\mathrm{dec}} \ll 5 \times 10^{14} \mathrm{~g}$, i.e., $M_{\mathrm{dec}} \approx 0$. The dashed, dot, dashed dot, and dashed dot dot lines correspond to $M_{\mathrm{dec}}$ values of $5 \times 10^{14} \mathrm{~g}, 10^{15} \mathrm{~g}, 10^{16} \mathrm{~g}, 10^{17} \mathrm{~g}$. The short dashed line corresponds to $M_{i}<M_{\mathrm{dec}}$, where such black holes trigger the vacuum decay immediately upon formation. 


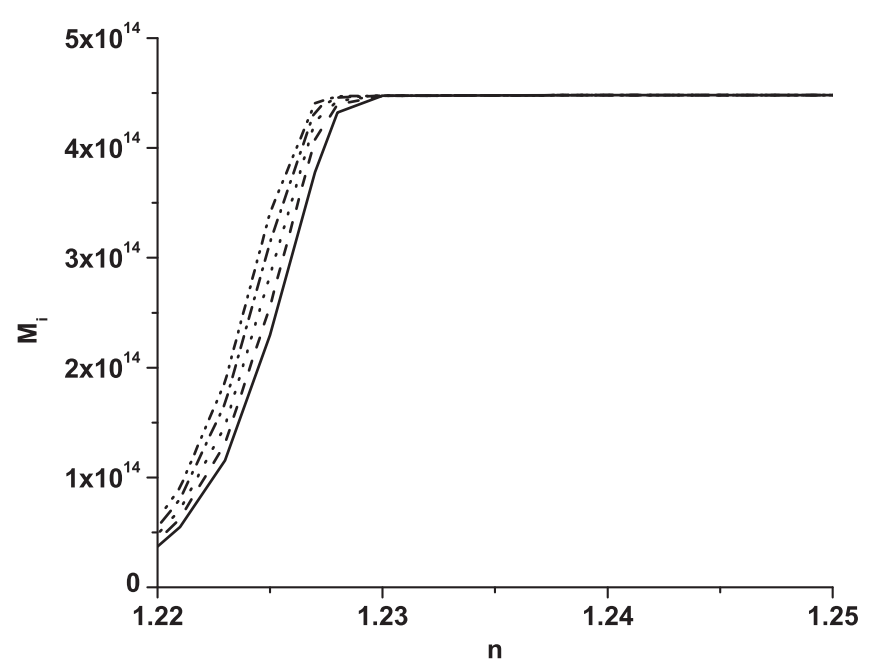

FIG. 6. This plot shows the primordial black hole masses (in grams) at the time of their formation, $M_{i}$, as a function of the spectral index, $n$. The solid lines represent the $\mathcal{P}=1$ value for the portion of the universe which already transitioned to the new vacuum at present time. Below this line we have $\mathcal{P}>1$ and the whole universe today would be destroyed. Above this line, we have $\mathcal{P}<1$, and the universe is safe. The dashed, dot, dashed dot and dashed dot dot lines represent the values $\mathcal{P}=10^{-1}$, $\mathcal{P}=10^{-2}, \mathcal{P}=10^{-3}$ and $\mathcal{P}=10^{-4}$ respectively, where the phase transition has not completed yet, but there are bubbles of true vacuum present in the universe.

\section{CONCLUSIONS}

We demonstrated here that it is possible to connect the parameters of the Higgs potential with the primordial black hole masses and physics of their formation (in our case the spectral index of perturbations that leads to their formation). We used the recent result that corrections due to black hole seeds can significantly increase the tunneling probability from the false to true Higgs vacuum. Any primordial black hole that had enough time to lose its mass from its formation till today to fit into an appropriate mass range for the corresponding choice of the Higgs potential parameters could trigger the false Higgs vacuum decay. If there is no new physics beyond the standard model, any black hole lighter than $4.5 \times 10^{14} \mathrm{~g}$ could trigger the decay. If some new physics beyond the standard model modifies the Higgs potential, then the black hole masses change appropriately. The decay rate is proportional to the exponential of the difference in entropy of the seed and remnant black holes masses, roughly $\left(M_{+}+\mu_{-}\right)\left(M_{+}-\mu_{-}\right)$. Numerically, this exponent varies slowly with $M_{+}$, and so can be regarded as $M \delta M$. The branching ratio that determines when the decay dominates evaporation is therefore most sensitive to the difference in seed and remnant masses that in turn is determined by an integral of the energy momentum of the scalar field bounce solution. Therefore, any potential that has a very thick bounce solution and correspondingly small $\delta M$ will have a much higher threshold of black hole mass for vacuum decay catalysis. We explored the threshold for a range of masses including the primordial black holes that could contribute to dark matter. Such black holes are outside the current SM parameter ranges, but could potentially be relevant for beyond the standard model potentials.

However, just triggering the decay is not enough to destroy the universe, and automatically exclude associated black hole range. For a successful completion of the first order phase transition the bubbles have to percolate, which in turn depends on the number density of the created bubbles. Since every black hole that can initiate the false vacuum decay will create a bubble, the number of the bubbles is equal to the number of such primordial black holes. We then trace evolution of the bubbles. The bubbles of the true vacuum expand with the speed of light, but the background universe expands as well, so their number density decreases. We define a quantity $\mathcal{P}$, which represents a portion of the universe which already transitioned to the new vacuum at the current time. For $\mathcal{P} \geq 1$, the universe is destroyed, and the associated range of parameters is excluded.

Our procedure can be used in two ways. If we use the Higgs potential parameters as an input, we can constrain the black hole masses and the physics of formation (e.g., the spectral index of perturbations). In turn, if we ever discover primordial black holes of definite mass, we can use it to constrain the Higgs potential parameters, or indeed the presence of extra dimensions [52,53].

\section{ACKNOWLEDGMENTS}

It is a pleasure to thank Ian Moss for helpful discussions. D. C. D. was supported by the National Science Foundation of China (Grants No. 11433001 and No. 11775140), National Basic Research Program of China (973 Program 2015 CB857001), and the Program of Shanghai Academic/Technology Research Leader under Grant No. 16XD1401600. R. G. is supported in part by the Leverhulme grant Challenging the Standard Model with Black Holes, in part by STFC consolidated grant No. ST/ P000371/1, and in part by the Perimeter Institute. D. S. was partially supported by the U.S. National Science Foundation, under Grant No. PHY-1820738. Research at Perimeter Institute is supported by the Government of Canada through the Department of Innovation, Science and Economic Development Canada and by the Province of Ontario through the Ministry of Research, Innovation and Science. 
[1] G. Aad et al. (ATLAS Collaboration), Combined search for the Standard Model Higgs boson using up to $4.9 \mathrm{fb}^{-1}$ of $p p$ collision data at $\sqrt{s}=7 \mathrm{TeV}$ with the ATLAS detector at the LHC, Phys. Lett. B 710, 49 (2012).

[2] S. Chatrchyan et al. (CMS Collaboration), Combined results of searches for the standard model Higgs boson in $p p$ collisions at $\sqrt{s}=7 \mathrm{TeV}$, Phys. Lett. B 710, 26 (2012).

[3] I. V. Krive and A. D. Linde, On the vacuum stability problem in gauge theories, Nucl. Phys. 117, 265 (1976).

[4] M. S. Turner and F. Wilczek, Is our vacuum metastable, Nature (London) 298, 633 (1982).

[5] M. Sher, Electroweak Higgs potentials and vacuum stability, Phys. Rep. 179, 273 (1989).

[6] G. Isidori, G. Ridolfi, and A. Strumia, On the metastability of the standard model vacuum, Nucl. Phys. B609, 387 (2001).

[7] G. Degrassi, S. Di Vita, J. Elias-Miro, J. R. Espinosa, G. F. Giudice, G. Isidori, and A. Strumia, Higgs mass and vacuum stability in the Standard Model at NNLO, J. High Energy Phys. 08 (2012) 098.

[8] A. Gorsky, A. Mironov, A. Morozov, and T. N. Tomaras, Is the Standard Model saved asymptotically by conformal symmetry? Zh. Eksp. Teor. Fiz. 147, 399 (2015) [JETP 120, 399 (2015)].

[9] F. Bezrukov and M. Shaposhnikov, Why should we care about the top quark Yukawa coupling? Zh. Eksp. Teor. Fiz. 147, 389 (2015) [J. Exp. Theor. Phys. 120, 335 (2015)].

[10] J. Ellis, Discrete Glimpses of the physics landscape after the Higgs discovery, J. Phys. Conf. Ser. 631, 012001 (2015).

[11] K. Blum, R. T. D’Agnolo, and J. Fan, Vacuum stability bounds on Higgs coupling deviations, J. High Energy Phys. 03 (2015) 166.

[12] S. Coleman, Fate of the false vacuum: Semiclassical theory, Phys. Rev. D 15, 2929 (1977).

[13] C. G. Callan and S. Coleman, Fate of the false vacuum II: First quantum corrections, Phys. Rev. D 16, 1762 (1977).

[14] S. Coleman and F. De Luccia, Gravitational effects on and of vacuum decay, Phys. Rev. D 21, 3305 (1980).

[15] I. Y. Kobzarev, L. B. Okun, and M. B. Voloshin, Bubbles in metastable vacuum, Yad. Fiz. 20, 1229 (1974) [Sov. J. Nucl. Phys. 20, 644 (1975)].

[16] R. Gregory, I. G. Moss, and B. Withers, Black holes as bubble nucleation sites, J. High Energy Phys. 03 (2014) 081.

[17] P. Burda, R. Gregory, and I. Moss, Gravity and the Stability of the Higgs Vacuum, Phys. Rev. Lett. 115, 071303 (2015).

[18] P. Burda, R. Gregory, and I. Moss, Vacuum metastability with black holes, J. High Energy Phys. 08 (2015) 114.

[19] P. Burda, R. Gregory, and I. Moss, The fate of the Higgs vacuum, J. High Energy Phys. 06 (2016) 025.

[20] L. Cuspinera, R. Gregory, K. Marshall, and I. G. Moss, Higgs vacuum decay from particle collisions? Phys. Rev. D 99, 024046 (2019).

[21] W. A. Hiscock, Can black holes nucleate vacuum phase transitions? Phys. Rev. D 35, 1161 (1987).

[22] V. Berezin, V. Kuzmin, and I. Tkachev, O(3) invariant tunneling in general relativity, Phys. Lett. B 207, 397 (1988).

[23] N. Tetradis, Black holes and Higgs stability, J. Cosmol. Astropart. Phys. 09 (2016) 036.
[24] P. Chen, G. Domènech, M. Sasaki, and D. h. Yeom, Thermal activation of thin-shells in anti-de Sitter black hole spacetime, J. High Energy Phys. 07 (2017) 134.

[25] D. Gorbunov, D. Levkov, and A. Panin, Fatal youth of the Universe: Black hole threat for the electroweak vacuum during preheating, J. Cosmol. Astropart. Phys. 10 (2017) 016.

[26] K. Mukaida and M. Yamada, False vacuum decay catalyzed by black holes, Phys. Rev. D 96, 103514 (2017).

[27] N. Oshita, M. Yamada, and M. Yamaguchi, Compact objects as the catalysts for vacuum decays, Phys. Lett. B 791, 149 (2019).

[28] K. Kohri and H. Matsui, Electroweak vacuum collapse induced by vacuum fluctuations of the Higgs field around evaporating black holes, Phys. Rev. D 98, 123509 (2018).

[29] E. Greenwood, E. Halstead, R. Poltis, and D. Stojkovic, Dark energy, the electroweak vacua and collider phenomenology, Phys. Rev. D 79, 103003 (2009).

[30] S. Hawking, Gravitationally collapsed objects of very low mass, Mon. Not. R. Astron. Soc. 152, 75 (1971).

[31] B. J. Carr and S. W. Hawking, Black holes in the early Universe, Mon. Not. R. Astron. Soc. 168, 399 (1974).

[32] M. Y. Khlopov, Primordial black holes, Res. Astron. Astrophys. 10, 495 (2010).

[33] B. Carr, F. Kuhnel, and M. Sandstad, Primordial black holes as dark matter, Phys. Rev. D 94, 083504 (2016).

[34] M. Zumalacarregui and U. Seljak, Limits on Stellar-Mass Compact Objects as Dark Matter from Gravitational Lensing of Type Ia Supernovae, Phys. Rev. Lett. 121, 141101 (2018).

[35] J. R. Espinosa, D. Racco, and A. Riotto, Cosmological Signature of the Standard Model Higgs Vacuum Instability: Primordial Black Holes as Dark Matter, Phys. Rev. Lett. 120, 121301 (2018).

[36] D. N. Page, Particle emission rates from a black hole, Phys. Rev. D 13, 198 (1976).

[37] B. J. Carr, J. H. Gilbert, and J. E. Lidsey, Black hole relics and inflation: Limits on blue perturbation spectra, Phys. Rev. D 50, 4853 (1994).

[38] B. J. Carr and J. H. MacGibbon, Cosmic rays from primordial black holes and constraints on the early universe, Phys. Rep. 307, 141 (1998).

[39] B. J. Carr, K. Kohri, Y. Sendouda, and J. Yokoyama, New cosmological constraints on primordial black holes, Phys. Rev. D 81, 104019 (2010).

[40] J. H. MacGibbon, T. N. Ukwatta, J. T. Linnemann, S. S. Marinelli, D. Stump, and K. Tollefson, Primordial black holes, arXiv:1503.01166.

[41] B. J. Carr, K. Kohri, Y. Sendouda, and J. Yokoyama, Constraints on primordial black holes from the Galactic gamma-ray background, Phys. Rev. D 94, 044029 (2016).

[42] A. M. Green and A. R. Liddle, Constraints on the density perturbation spectrum from primordial black holes, Phys. Rev. D 56, 6166 (1997).

[43] H. Kodama, M. Sasaki, and K. Sato, Abundance of primordial holes produced by cosmological first order phase transition, Prog. Theor. Phys. 68, 1979 (1982).

[44] M. Crawford and D. N. Schramm, Spontaneous generation of density perturbations in the early universe, Nature (London) 298, 538 (1982).

[45] S. D. H. Hsu, Black holes from extended inflation, Phys. Lett. B 251, 343 (1990). 
[46] J. L. Sanz and L. J. Giocoechea, Observational and theoretical aspects of relativistic astrophysics and cosmology, Proceedings, International Course, Santander, Spain, 1984 (World Scientific, Singapore, 1985), p. 352.

[47] T. Harada, C. M. Yoo, and K. Kohri, Threshold of primordial black hole formation, Phys. Rev. D 88, 084051 (2013); Erratum, Phys. Rev. D 89, 029903 (2014).

[48] A. R. Liddle and D. H. Lyth, The cold dark matter density perturbation, Phys. Rep. 231, 1 (1993).

[49] T. Bringmann, C. Kiefer, and D. Polarski, Primordial black holes from inflationary models with and without broken scale invariance, Phys. Rev. D 65, 024008 (2001).
[50] H. I. Kim, C. H. Lee, and J. H. MacGibbon, Diffuse gammaray background and primordial black hole constraints on the spectral index of density fluctuations, Phys. Rev. D 59, 063004 (1999).

[51] N. Aghanim et al. (Planck Collaboration), Planck 2018 results. VI. Cosmological parameters, arXiv:1807.06209.

[52] K. J. Mack and R. McNees, Bounds on extra dimensions from micro black holes in the context of the metastable Higgs vacuum, Phys. Rev. D 99, 063001 (2019).

[53] L. Cuspinera, R. Gregory, K. M. Marshall, and I. G. Moss, Higgs Vacuum decay in a braneworld, Int. J. Mod. Phys. D 29, 2050005 (2020). 